\title{
A Reliability-Based Optimization Scheme for Maintenance Management in Large-Scale Bridge Networks
}

\author{
Xiaofei $\mathrm{Hu}$ \\ Ph.D., Dept. of Civil and Environmental Engineering, University of California, Berkeley, CA, U.S. \\ Carlos Daganzo \\ Professor, Dept. of Civil and Environmental Engineering, University of California, Berkeley, CA, U.S. \\ Samer Madanat \\ Professor, Dept. of Civil and Environmental Engineering, University of California, Berkeley, CA, U.S.
}

ABSTRACT: Incorporating network topologies in bridge management problems is computationally difficult. Because of the interdependencies among bridges in a network, they have to be analyzed together. Simulation-based numerical optimization techniques adopted in past research are limited to networks of moderate sizes. In this paper, a simple framework is developed to determine optimal maintenance plans for large networks with many bridges. The objective is to minimize disruption, specifically, the extra travel distance caused by potential bridge failures over a planning horizon and under a budget constraint. It is conjectured and then verified that the expected increase in vehicle-miles travelled due to failures can be approximated by the sum of expected increases due to individual failures. This allows the network-level problem to be decomposed into single-bridge problems and tackled efficiently. The computational effort increases linearly with the number of bridges.

\section{INTRODUCTION}

It is widely acknowledged that the social and economic development of any region relies on satisfactory performance of their transportation networks. In particular, highway bridges represent the vulnerable components of highway networks. Most highway bridges are built at the connections to on/off ramps, the intersections of highways, and the crossings over rivers or railways. Bridge closures can sever effective links connecting origins and destinations. Vehicles need to bypass the unserviceable links, which can increase total travel time and distance.

Bridges deteriorate progressively over their lifetime due to environmental effects and traffic loads. This paper's scope is bridge failures as the unserviceability of bridge structures caused by gradual deterioration. Taking bridge decks as an example, the major mechanism of concrete deck failure is spalling, which follows the corrosion of the steel rebars and delamination cracking in the concrete. Corrosion starts when chlorides reach the level of steel. Rust builds up around the rebars; the volume of steel expands. The expansion causes concrete fractures and the cohesion between concrete and steel gradually vanishes. The structural resistance of the bridge deck decreases, and it may eventually result in the deck's failure (i.e., the occurrence of spalling).

The problem is important because the current conditions of federal bridges are unsatisfactory, and maintenance and repairs budgets are limited. According to ASCE (2013), the average age of bridges in the U.S. is 42 years old; more than $30 \%$ of existing bridges have exceeded their 50-year design life. Timely and adequate maintenance activities must be carried 
out to ensure safety and satisfactory service levels. Faced with an \$8 billion annual investment shortfall for federal bridges, it is necessary to allocate and distribute the limited maintenance resources in an optimal manner.

There is a growing body of literature that addresses the optimal maintenance planning for individual bridges or systems of bridges (Markow et al., 1993; Frangopol et al., 2001; Kong and Frangopol, 2003; Robelin and Madanat, 2007; Robelin and Madanat, 2008). Since maintenance policies are usually made by agencies responsible for an entire highway network, research has expanded to networks with multiple bridges. Network-level problem formulations usually seek maintenance policies that best balance network performance and agency cost. The former has been frequently quantified by graph-theoretic indicators of network reliability or connectivity. The latter is usually quantified by the discounted life-cycle maintenance cost. Liu and Frangopol (2005, 2006) applied a genetic algorithm and a network connectivity metric for a single O/D pair to solve this problem. Bocchini and Frangopol (2013) and $\mathrm{Hu}$ and Madanat (2014) expanded it to multiple O/D pairs.

Although connectivity is the fundamental function of a network, it is an inaccurate proxy for user cost, which is more closely related to time and distance travelled. Some researchers have adopted travel time as the criterion. Orcesi and Cremona (2010) developed a bridge network maintenance framework for a Pareto-improving optimization of MR\&R activities. Bocchini and Frangopol (2010) introduced an indicator associated with the total travel time and total travel distance of the network.

The network-level problem is difficult to solve, because the extra time and distance due to a bridge failure depend on whether other (nearby) bridges have failed. Thus, all bridges in a network have to be analyzed together. Works that include realistic metrics for user cost, e.g., Bocchini and Frangopol (2010), invariably resort to simulation-based numerical optimization algorithms, such as genetic algorithms, which do not guarantee convergence to optimality, and are computationally expensive. As a result, only networks of moderate sizes (10-30 bridges) can be tackled. However, most real networks include hundreds, if not thousands of bridges; for example, there are about 6,000 bridges in the San Francisco Bay Area.

This paper presents a solution method for bridge management problems of realistic sizes, using a realistic user cost metric. It is assumed that the network is not congested. Thus, user costs can be accurately reflected by the extra vehicle-miles travelled (VMT) caused by the closure of bridges. The paper is organized as follows. The problem is defined in section 2. A simplifying conjecture is proposed and verified in section 3. With this conjecture, a Lagrangian decomposition method for the network optimal management is presented in section 4. Finally, some conclusions and directions for future research are presented in section 5 .

\section{PROBLEM DEFINITION}

The bridge network considered in this paper consists of bridges and roads that connect these bridges. Bridges are the only vulnerable components of the network, and they are managed by a single agency such as a state department of transportation.

Because the deck is the bridge component that deteriorates fastest and requires the largest maintenance budget, only deck condition is considered in this paper. This condition is represented by a deck's reliability index $\beta$. This index is related to the deck's failure probability in a time interval between inspections by the probit transformation; so that $\Phi(-\beta)$ is said probability, where $\Phi$ is the standard normal cumulative distribution function.

Given a set of actions, $\vec{a}$, and a parameter vector $\pi$ that characterizes the bridge itself (such as its design, material type, traffic, and environment), the deck reliability index $\beta(t \mid \pi, \vec{a})$ is a stochastic process over time. It is reasonably assumed that this deterioration 
process $\beta(t \mid \pi, \vec{a})$ is independent across bridges, because correlations in performance across bridges due to the environment and traffic are captured by the parameter vector $\pi$.

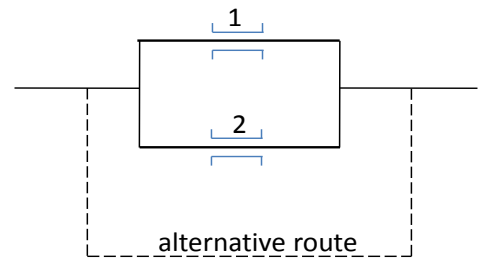

(a)

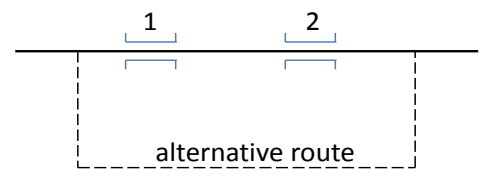

(b)

Figure 1: The effect of bridge failures on VMT

Bridge failures can sever efficient paths connecting origins and destinations, increasing travel cost. In the absence of congestion, the link user costs and the shortest paths connecting origins and destinations are fixed. The link cost for one trip can be reasonably expressed as a linear combination of the fixed link distance and link time. The cost of a set of failures can be measured by the difference in the travel costs of the users, assuming that they always choose the cheapest paths. This can be measured by calculating the difference in the costs before and after bridge failures for each $\mathrm{O} / \mathrm{D}$ pair, multiplying each difference by the O/D flow and adding the results for all O/D pairs. This process is necessary because when several bridges fail simultaneously, the cost is not necessarily the sum of costs associated with individual failures. Figure 1 illustrates the point. The alternative routes in both (a) and (b) represent costly paths. For case (a), the cost of a joint failure is much greater than that of a single failure, since vehicles have to take the costly alternative route when both bridges fail. On the other hand, for case (b), the joint failure cost is the same as the cost of a single failure. However, if the two failing bridges are far apart compared to a trip's length, then every O/D pair is affected by at most one bridge. Obviously, in this case, the added cost for two failures is the sum of the added costs for the single failures. It is shown in the next section that if the failure probabilities are sufficiently low, these close failures are so unlikely that the added total cost can be accurately approximated by the sum of the costs for individual failures.

\section{CONJECTURE AND VERIFICATION}

In this section, we show when the increase in cost for an entire network is approximately equal to the sum of the increases caused by individual failures. There are two conditions for this to happen. The first is that the failure rate of bridges is very low, and thus the circumstance that close bridges fail simultaneously is rare. The second condition is that the networks should be redundant; there must be alternative routes available for vehicles to reach their destination even if one or more links are out of service. These two conditions hold for developed regions, where highway facilities are dense and maintained regularly.

The conjecture is now verified. Section 3.1 proves it analytically for a class of homogeneous networks, and section 3.2 verifies it in a real network. The networks are assumed to be uncongested, and users always choose the minimum-cost paths.

\subsection{Analytic proof for homogeneous grid networks}

We choose idealized grids to prove the conjecture because they can represent very different scenarios by adjusting just a few parameters and can be modeled analytically. Section 3.1.1 describes the features and parameters of the network. Section 3.1.2 presents a formula for the error bound, and shows that the error is negligibly small.

\subsubsection{Network description and notations}

The region under analysis is a square covered by a grid network with $\mathrm{M}$ arterials in each direction (north-south and east-west), as shown in Figure 2. This grid is overlaid over an infinitely dense 
grid of slower streets, which are used to access the arterials. The length of an arterial block is $L$. Bridges are located at intersections; there are $\mathrm{m}$ blocks between two neighboring bridges. The bridge failure probability is $p$, and the failures happen independently. With no loss of generality, we assume that all bridges are in the north-south direction. Thus, when a bridge fails, vehicles on the corresponding north-south arterial cannot go through the bridge, and afterwards vehicles cannot make turning movements at the intersection either, they can travel east-west.

It is assumed that trip origins are uniformly distributed, that all trips have a maximum length of $n L$, and that for each origin, destinations are uniformly distributed within the origin's reachable area. The demand density is $q$ $\left(\mathrm{veh} / \mathrm{m}^{4}\right)$, representing the number of vehicles generated from a unit area and attracted to a unit area in the reachable area.

Vehicles access and egress the network at the arterial intersections closest to their origins and destinations. Because trips normally span many blocks (i.e. $\mathrm{n}$ is typically large), only vehicle-miles travelled on the network are considered; the access/egress distances are neglected.

\subsubsection{Approximation formula and error bounds}

As shown in Figure 2, when a bridge fails, vehicles going from area A to area B (or vice versa) need to take neighboring arterials to reach their destinations. These vehicles have to travel to the neighboring streets and then travel back, resulting in an additional distance of $2 L$ per vehicle. All other O/D pairs remain unaffected. Thus, the increase in VMT due to the failure, denoted $\omega$, is the product of $2 L$ and the total number of vehicles affected by the failures:

Number of affected vehicles

$$
=2 \cdot q \cdot \int_{0}^{n L}(n L-x) L \cdot L d x=n^{2} L^{4} q .
$$

Thus,

$$
\omega=n^{2} L^{4} q \times 2 L=2 n^{2} L^{5} q .
$$

Now, use $\Omega$ to represent the expected increase in VMT of the entire network and $\Omega^{\prime}$ the expected increase under the conjecture. Since there are $M^{2} / \mathrm{m}^{2}$ bridges in the network, we have:

$$
\Omega^{\prime} \approx p\left(\frac{M^{2}}{m^{2}}\right) \omega=\frac{2 p n^{2} L^{5} q M^{2}}{m^{2}}
$$

This formula is approximate because it uses (1) and (2) for failures close to the boundary, even though the boundary then reduces the number of affected vehicles. The formula is asymptotically exact as $M \rightarrow \infty$ and should be quite accurate for large networks; i.e. when $M / n \gg 1$.

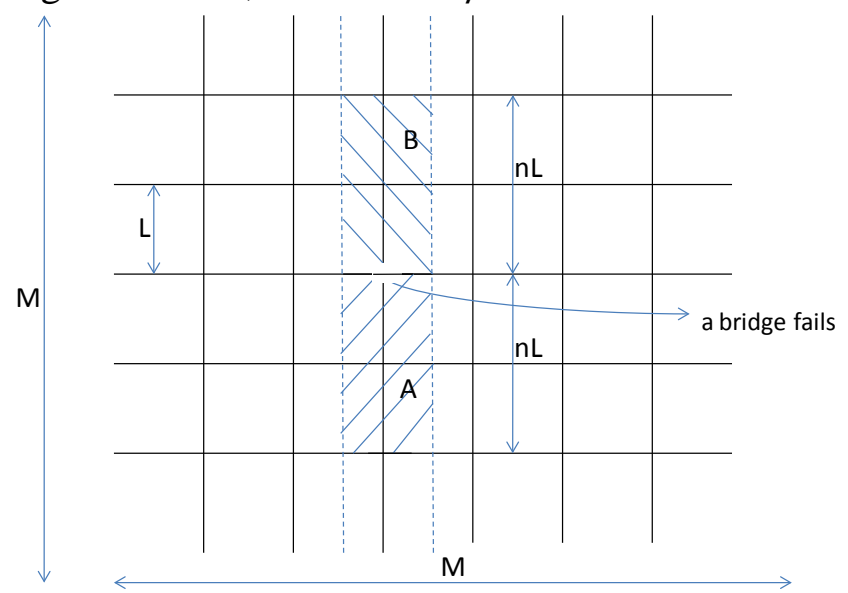

Figure 2: Illustration of an individual failure

The approximation error $E$ is defined as the expected value of the absolute difference between the exact increase in vehicle-miles travelled due to simulated failures and the approximate increase derived by summing the increases obtained for each failed bridge in isolation from the others. To assess the error, we investigate the interaction effect between two close failures, and then derives error bounds for cases involving more failures. Finally, these results are synthesized to obtain an overall error bound. It turns out that $E$ is negligibly small. To quantify it, let $E^{\prime}=E / \Omega$ be the relative error. It is proved that $E^{\prime} \leq B$, where

$$
\begin{aligned}
& B=n p+13(6 n p)^{2}, \text { if } m=1 \\
& B=\frac{n p}{3 m}+8\left(\frac{n p}{m}\right)^{2}, \text { if } m \geq 2
\end{aligned}
$$


Since the right sides of these equations tend to 0 as $n / m \rightarrow 0$, this establishes that (3) is a very good approximation in practical cases, where typically $p \sim 10^{-5}$ and $n / m \sim 10^{1}$. The detailed proof of Equations (4) is provided in $\mathrm{Hu}$ et al. (2014).

Equations (4) can be explained qualitatively as follows. For a given bridge failure, there are $O(n / m)$ bridges nearby that can interact with it. If $n p / m \ll 1$, it is very unlikely that close failures occur, and therefore, their interactions are negligible. This should also be true if the network and the demand are inhomogeneous. Thus, the conjecture should also hold for real networks.

\subsection{Verification in real world network}

Although many networks do not have exact grid layouts, they can be obtained by deforming a grid. Therefore, equations (4) are now reexpressed in terms of variables that can be measured for real networks. The accuracy of this approximation is then verified using the highway network of the San Francisco Bay Area.

To re-express equations (4), we need to replace $n$ and $m$ by equivalent parameters that would be meaningful for an arbitrary network. Since $\mathrm{n}$ and $\mathrm{m}$ are proxies for "trip length" and “distance between bridges”, let us introduce $L_{a}$ as the average trip length, and $D_{a}$ as the average distance between neighboring bridges. In a grid, $n=3 L_{a} / 2 L$, and $m=D_{a} / L$. Thus, (4b) can be rewritten in terms of the more generic parameters $L_{a}$ and $D_{a}$ as:

$$
B=\frac{L_{a} p}{2 D_{a}}+18\left(\frac{L_{a} p}{D_{a}}\right)^{2}, \text { if } \frac{D_{a}}{L} \geq 2
$$

Equation (4a) is not included because in most cases $D_{a} \gg L$.

According to the National Bridge Inventory, there are 6,107 bridges in the Bay Area, 549 of which are structurally deficient. Given the total area of the Bay Area, $D_{a}$ is estimated to be 1.07 miles. The original highway network is adopted from the MTC (Metropolitan Transportation Commission) travel model. The traffic demand comes from MTC's demand forecasts for year
2010, from 6 a.m. to 10 a.m. on a weekday. Dividing total vehicle-miles travelled by the number of trips, $L_{a}$ is estimated to be 5.72 miles.

Simulations were carried out to evaluate approximation errors in the real network and in an equivalent grid. The failure probability of deficient bridges was set to be $1.0 \times 10^{-3}$, corresponding to a low reliability index of 3 . Other bridges have a smaller failure rate of $3.0 \times 10^{-4}$, although in reality $p \sim 10^{-5}$. For each of the ten instances generated, the failures were recorded, as was the added user cost. The increase in VMT due to a set of failures was then compared with the sum of the increases in VMT due to single failures to determine the absolute error. These absolute errors were then averaged across the 10 instances to estimate the absolute error, $E_{s}$, and the relative error, $E_{s}{ }^{\prime}$.

For the equivalent grid network, $n / m=$ $3 L_{a} / 2 D_{a} \approx 8$. The absolute error, $E_{g}$, and the relative error, $E_{g}{ }^{\prime}$, were estimated by averaging 200 instances on a grid with $n=16, m=2$. The estimated relative errors from simulation, $\widehat{E_{S}^{\prime}}$ and $\widehat{E_{g}}{ }^{\prime}$, and the error bound from formula (5), B, are shown in Table 1 . The table shows that the analytic bound also holds for the real networks, and more importantly that the results for the real and idealized networks are very close. This supports that the conjecture can be used with confidence in real networks.

Table 1: Comparison of the mean errors and the error bound

\begin{tabular}{|l|l|c|}
\hline Mean Relative & Mean Relative & Error bound \\
Error in the & Error in the & $B(\%)$ \\
Real Network & Equivalent & \\
$\widehat{E_{S}^{\prime}}(\%)$ & Grid $\widehat{E_{g}^{\prime}}(\%)$ & \\
\hline $6.42 \times 10^{-2}$ & $4.96 \times 10^{-2}$ & $1.04 \times 10^{-1}$ \\
\hline
\end{tabular}

Although the example used a distance metric, similar results would have been obtained with any reasonable linear combination of link distance, time and toll. After all, these changes could be calculated into equivalent distances, which may change the shape of the network; and we have seen from the first two columns of 
Table 1 that these changes have insignificant effects on accuracy.

\section{NETWORK-LEVEL OPTIMIZATION}

Under the conjecture, the total user cost of the network can be explicitly expressed as the sum of user costs induced by individual bridges. The network problem can then be broken down into smaller subproblems using Lagrangian decomposition. The subproblems can be tackled with dynamic programming. In the second part of this section, the method is applied to a virtual network of 6,000 bridges as an illustration.

\subsection{Formulation and Methodology}

The network-level maintenance management problem solves for the optimal maintenance policy that minimizes the expected increase in user cost due to possible bridge deck failures, subject to a multiyear budget constraint. Solving the problem under different budgets yields a Pareto frontier of user costs and agency costs. There are a range of maintenance actions that can be taken, and maintenance decisions are made every year for each bridge deck. The current condition of each deck is assumed to be known perfectly, meaning that inspections are carried out every year and are error free.

There are $\mathrm{N}$ bridges in the network, and the planning horizon is $\mathrm{T}$ years. The set of decision variables is $\left\{a_{i t}\right\},(i=1,2, \ldots, N ; t=1,2, \ldots, T)$, whose elements are the actions applied on the bridges in each year. The failure probability of bridge $i$ in year $t, p_{i t}$, depends on the initial conditions and the history of maintenance actions. Therefore, $p_{i t}$ is a function of time t, initial condition $p_{i 0}$, and the history of maintenance actions $\overrightarrow{a_{l t}}=\left\{a_{i 1}, a_{i 2}, \ldots, a_{i t}\right\}$. Under the conjecture, the increase in user cost in the network is approximately equal to the sum of the increases in costs due to individual failures. The formulation of the optimization problem is then:

$$
\min _{a_{i t} \in A} \sum_{i=1}^{N} \sum_{t=1}^{T} \Delta_{i}(t) \cdot p_{i t}\left(p_{i 0}, \overline{a_{l t}}\right)
$$

$$
\text { s.t. } \sum_{i=1}^{N} \sum_{t=1}^{T} c_{i}\left(a_{i t}\right) \leq b
$$

A - set of all possible MR\&R actions, including maintenance, repair, and reconstruction activities, and do-nothing; $\Delta_{i}(t)$ - increase in user cost due to the failure of bridge deck i in year $t$; $c_{i}\left(a_{i t}\right)$ - the maintenance cost of action $a_{i t}$ on bridge deck $i$ at year $t$;

$b$ - total budget.

This problem can be tackled using dual decomposition. The dual problem to the original optimization is: $\max _{\lambda} \sum_{i=1}^{N} g_{i}(\lambda)-\lambda b$, where $\lambda$ is the Lagrangian multiplier, and subprorblems $g_{i}(\lambda)$ is to solve for $a_{i t}$ 's that minimize $\sum_{t=1}^{T}\left(\Delta_{i}(t) \cdot p_{i}\left(t, p_{i 0}, \overrightarrow{a_{t t}}\right)+\lambda \cdot c_{i}\left(a_{i t}\right)\right)$, for a given $\lambda$. The first order condition for the dual problem is $\sum_{i=1}^{N} \sum_{t=1}^{T} c_{i}\left(a_{i t}\right)=b$, and simple algorithms, such as bisection method can be used to update $\lambda$.

The subproblems $g_{i}(\lambda)$ are formulated and solved as Markov decision processes with augmented states. In traditional Markovian models with bridge component condition as the state, the history of the condition is not taken into account, which is seen as a limitation. According to Robelin and Madanat (2007), the effects of the history of deterioration and maintenance can be captured by a threedimensional vector $x=(\beta, u, \tau)$, where $\beta$ is the reliability index, $u$ represents the type of the latest action performed on the bridge deck, and $\tau$ represents the time since the latest action. Therefore, the deterioration process can be modeled as Markovian, using vector $x$ as the state.

\subsection{Numerical example}

The numerical study is implemented on a network with 6,000 bridges. To carry out lifecycle analysis of bridge networks, the planning horizon is set to be 60 years. The bridges are randomly divided into 10 homogeneous groups to represent heterogeneity in the network. The transition probabilities 
matrices are obtained by Monte Carlo simulation, based on the stochastic lifetime profile of the reliability index from Frangopol et al. (2001). Details of this Monte Carlo simulation process used are provided in Robelin and Madanat (2007). Four actions are considered, do-nothing, maintenance, repair and reconstruction. The costs of the maintenance actions are adopted from Kong and Frangopol (2003). Our example uses a distance-based metric, and the increase in VMT caused by a bridge failure is estimated based on the traffic demand and the transportation network of the San Francisco Bay Area. To capture the heterogeneity across different groups, the parameters provided in these papers are adjusted within realistic ranges.

The algorithm is implemented to solve the optimization for different budgets. Figure 3 plots for different budget levels, the inverse of $\lambda$, which represents the extra budgetary dollars required to save one additional vehicle-mile of user "cost" (\$/veh-mile). As expected, as the budget increases, the cost of saving an additional vehicle-mile increases, meaning that the better the system, the more costly it is to find extra savings.

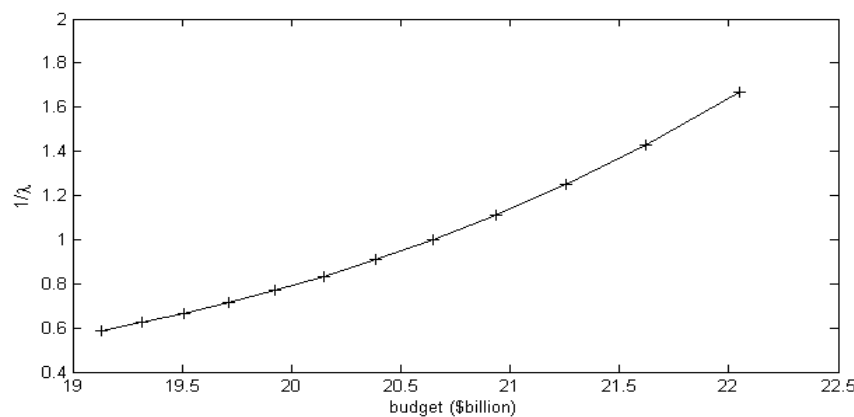

Figure 3: Numerical implementation for 6,000 bridges

Figure 3 also shows that the budget required to achieve optimality depends on how much the highway agency values one vehicle-mile of user cost. In our example, if this monetary value is $\$ 1$, then the budget required for the network's lifecycle maintenance is around $\$ 20.6$ billion.

For a prescribed value of user costs, the method can yield the optimal maintenance policies for individual bridges. With $1 / \lambda$ set to be $\$ 1 /$ veh-mile, Figure 4 plots the expected amount of budget consumed by the three different maintenance actions at each year during the lifecycle. In our example, most of the construction actions are conducted between year 15 to year 40, and most of the repairs are conducted in the later period. This type of analysis can help agencies plan the maintenance budget over the lifecycle of bridge networks.

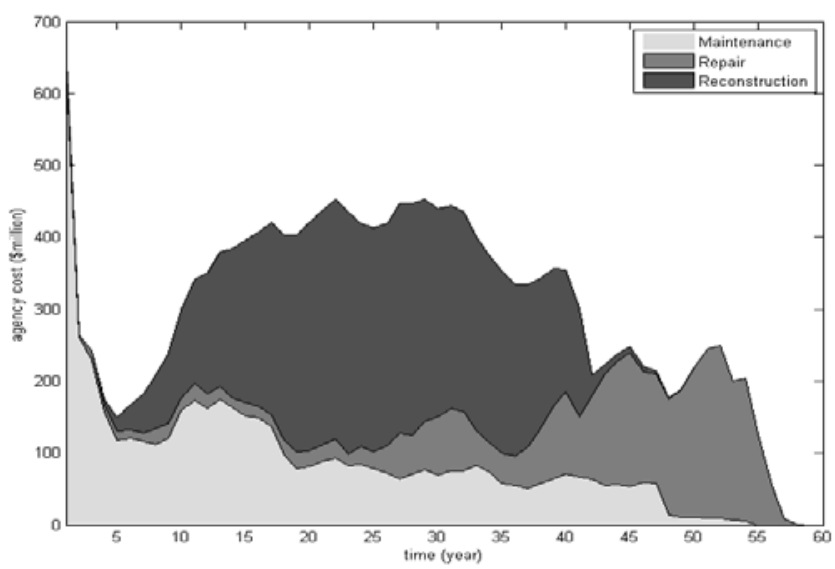

Figure 4: Lifecycle budget allocated on different maintenance actions

\section{CONCLUSION}

This paper shows how to find the optimal maintenance plans for deteriorating bridges in large-scale networks. The objective is to minimize the expected increase in user costs caused by potential bridge failures over a planning horizon, under a budget constraint. The main contributions are:

- The overall user cost is measured directly by the increase in travel time and distance, which are more relevant than connectivity or other metrics used in past research.

- It is verified that if a network is uncongested then the expected increase in user costs due to bridge deck failures can be approximated by the sum of increases due to individual failures. As a result, the network-level optimization problem can be decomposed.

- An efficient approach is developed, capable of optimizing the maintenance activities of 
large networks with thousands of deteriorating bridges over multiple periods.

These contributions pertain to uncongested networks. They apply because bridges separated by large distance do not interact. In the real world, there can be congested links, especially in urban areas during peak hours. Although congestion can enlarge the critical distance above which two bridges do not interact, the enlargement should be small for moderately congested networks. Thus, bound (4) should still hold in moderately congested networks, if one uses the congested link costs of the basic network (without failures) as the basis for evaluating the $\Delta_{i}(t)$ of the proposed methodology. If congestion is more severe, (4) can be violated, but it should be remembered that the bound is very tight. Thus, the method proposed in this paper should yield reasonable results even under typical congestion levels found in real cities. Since, to date, there are no alternative solution methods for large networks, the proposed methodology can serve as a benchmark against which improved methods can be compared.

In addition to congestion, further research should consider constraints on reliability for individual bridges for safety reasons, and user costs due to maintenance actions.

\section{REFERENCES}

ASCE, 2013 ReportCard on America's Infrastructure, www.infrastructurereportcard.org.

Bocchini, P. and Frangopol, D. M. (2011), “A Probabilistic Computational Framework for Bridge Network Optimal Maintenance Scheduling”, Reliability Engineering and System Safety, 96(2): 332 - 349.

Bocchini, P. and Frangopol, D. M. (2013), "Connectivity-Based Optimal Scheduling for Maintenance of Bridge Networks", Journal of Engineering Mechanics, 139(6): 760 - 769.

Frangopol, D. M., Kong, J. S. and Gharaibeh, E. S. (2001), "Reliability-Based Life-Cycle Management of Highway Bridges", Journal of Computing in Civil Engineering, ASCE; 15(1): $27-47$.
Hu, X. and Madanat, S. M. (2014), "Determination of Optimal MR\&R Policies for Retaining LifeCycle Connectivity of Bridge Networks", Journal of Infrastructure Systems, accepted.

Hu, X., Daganzo, C. F. and Madanat, S. M. (2014), "A Reliability-Based Optimization Scheme for Maintenance Management in Large-Scale Bridge Networks", Transportation Research Part $C$, under review.

Kong, J. S. and Frangopol, D. M. (2003), "Life-Cycle Reliability-Based Maintenance Cost Optimization of Deteriorating Structures with Emphasis on Bridges", Journal of Structural Engineering, 129(6): 818 - 828.

Liu, M. and Frangopol, D. M. (2005), "Balancing Connectivity of Deteriorating Bridge Networks and Long - Term Maintenance Cost through Optimization”, Journal of Bridge Engineering, 10(4): 468 - 481.

Liu, M. and Frangopol, D. M. (2006), "Optimizing Bridge Network Maintenance Management under Uncertainty with Conflicting Criteria: Life-Cycle Maintenance, Failure, and User Costs”, Journal of Structural Engineering, 132(11): 1835 - 1845.

Markow, M. J., Madanat, S. M., and Gurenich, D. I. (1993), "Optimal Rehabilitation Times for Concrete Bridge Decks”. Transportation Research Record 1392.

Newell, G. F. (1993), "Flow Around Distortions in A Dense Rectangular Grid Road Network, I. Theory, II. Examples", Proc. 12th Int. Symposium on the Theory of Traffic Flow and Transportation, Elsevier, 1993, pp. 1-16, 1736.

Orcesi, A. D., and Cremona, C. F. (2010), “A Bridge Network Maintenance Framework for Pareto Optimization of Stakeholders/Users Costs”, Reliability Engineering and System Safety, 95(11): $1230-1243$.

Robelin, C. A. and Madanat, S. M. (2007), "HistoryDependent Bridge Deck Maintenance and Replacement Optimization with Markov Decision Processes”, Journal of Infrastructure Systems, 13(3): 195 - 201.

Robelin, C. A. and Madanat, S. M. (2008), "Reliability-Based System-Level Optimization of Bridge Maintenance and Replacement Decisions”, Transportation Science, 42(4): 508 $-513$. 